\title{
Role of salt-inducible kinase 2 in the malignant behavior and glycolysis of colorectal cancer cells
}

\author{
XIAOHONG $\mathrm{NI}^{1}$, YONGJIANG FENG ${ }^{1}$ and XIANGWEI FU ${ }^{2}$ \\ ${ }^{1}$ Department of Gastrointestinal Surgery, Yancheng Dafeng People's Hospital, Yancheng, \\ Jiangsu 224100; ${ }^{2}$ Department of General Surgery, The Second Affiliated Hospital of \\ Hainan Medical University, Haikou, Hainan 570105, P.R. China
}

Received June 1, 2021; Accepted July 28, 2021

DOI: $10.3892 / \mathrm{mmr} .2021 .12460$

\begin{abstract}
Colorectal cancer (CRC) is the third most common type of cancer worldwide. Currently, surgery, chemotherapy and radiation therapy are the conventional approaches used to treat CRC. However, these therapy strategies cause several side effects. The present study aimed to develop an alternative and more effective treatment approach for patients with CRC. It has been reported that salt-inducible kinase 2 (SIK2) acts as an oncogene. Therefore, in the present study, the expression levels of SIK2 were determined in CRC cells using western blot analysis and reverse transcription-quantitative PCR. In addition, SIK2 was knocked down in CRC cells to evaluate its role in cell proliferation, migration, invasion and glycolysis using Cell Counting Kit-8, wound healing, Transwell assays and glycolysis cell-based assay kit, respectively. Additionally, the target genes of SIK2 were identified using bioinformatics analysis, while SIK2 overexpression experiments were carried out to determine whether SIK2 could regulate CRC cell malignant behavior and glycolysis. The results revealed that SIK2 was upregulated in CRC cells. Furthermore, SIK2 knockdown attenuated CRC cell proliferation, migration, invasion and glycolysis. Bioinformatics analysis predicted that SIK2 could interact with tripartite motif containing 28 (TRIM28), while TRIM28 overexpression could reverse the effects of SIK2 silencing on cell proliferation, migration, invasion and glycolysis. This finding indicated that the aforementioned effects of SIK2 were mediated by regulating TRIM28. In conclusion, the findings of the present study suggested that SIK2 may be involved in CRC carcinogenesis and glycolysis by regulating TRIM28 expression. These findings could provide a novel approach to targeted therapy and clinical diagnosis of $\mathrm{CRC}$ in the future.
\end{abstract}

Correspondence to: Professor Xiangwei Fu, Department of General Surgery, The Second Affiliated Hospital of Hainan Medical University, 368 Yehai Avenue, Haikou, Hainan 570105, P.R. China E-mail: fuxiangwei12@126.com

Key words: salt-inducible kinase 2, tripartite motif containing 28, colorectal cancer, glycolysis, malignancy

\section{Introduction}

Colorectal cancer (CRC) is a heterogeneous disease of the intestinal epithelium, which is characterized by the accumulation of mutations and immune response disorders (1). CRC is the third most common type of cancer worldwide, with an incidence rate of $10.2 \%$ among all cancer types and a mortality rate of $9.2 \%$ (2). Its prevalence has been gradually increasing among individuals $<50$ years old (1), while the survival rate of patients with metastasis is estimated to be only $18.5 \%$ in the United States (3). Although early diagnosis via fecal occult blood testing and colonoscopy can reduce the mortality rate to a certain extent, the mortality rate of CRC remains high (4). The currently available conventional methods for treating CRC are surgery, chemotherapy and radiation therapy. These therapeutic approaches may be used alone or in combination, based on the progression of CRC during treatment (5-7). In addition, for localized, easily accessible CRC, total mesenteric rectal resection is considered as an effective treatment approach (8-10). In mesenteric rectal resection, the cancer tissue is removed via laparoscopic and transanal surgery, followed by adjuvant chemotherapy or radiotherapy (11). However, these therapies are non-specifically cytotoxic to all rapidly dividing cells, inevitably causing several side effects (12). Following neoadjuvant therapy, 54\% of patients with CRC develop disease relapse (13). Therefore, developing alternative and more effective therapeutic strategies for treating patients with $\mathrm{CRC}$ is crucial.

It is estimated that there will be 2.2 million new CRC cases and 1.1 million CRC-related deaths by 2030 worldwide (14). Biomarkers are objective indicators that can be isolated or measured in the human body and used to evaluate the pathological process of cancer or predict treatment effectiveness. In addition, biomarkers can be used as tools for the early detection and personalized therapy for CRC $(15,16)$. More specifically, biomarkers provide information in order to evaluate the progression or recurrence of the disease at different stages and provide guidance for individualized treatment. For asymptomatic patients, biomarkers can be used for the diagnosis of early CRC and premalignant lesions, to achieve favorable survival (17). For patients with advanced disease, biomarkers can be used to predict disease progression, including the risk of recurrence and survival $(16,18)$. Ongoing advances in the 
molecular classification of CRC subtypes, DNA methylation and microRNA biogenesis have resulted in the identification of numerous biomarkers for CRC (19). Therefore, further exploration and mechanistic research on biomarkers in human cancer may prove to be of great diagnostic and prognostic value.

It has been reported that salt-inducible kinase 2 (SIK2) is upregulated in breast (20), ovarian (21) and cervical cancer (22), and promotes cancer progression, thus suggesting that SIK2 may act as an oncogene. To the best of our knowledge, the role of SIK2 in CRC has not been previously reported. Therefore, the present study aimed to reveal the effect of SIK 2 on CRC cell lines and further examine its underlying mechanism of action. The results of the current study may provide a novel approach to the targeted therapy and clinical diagnosis of CRC.

\section{Materials and methods}

Cell lines and transfection. The normal colonic epithelial cell line, NCM460, and the CRC cell lines, LoVo, SW480, SW620, CW2 and HCT116, were purchased from the National Infrastructure of Cell Line Resource (Beijing, China). Each cell line was cultured in DMEM (Gibco; Thermo Fisher Scientific, Inc.) supplemented with $10 \%$ FBS and $100 \mathrm{U} / \mathrm{ml}$ penicillin-streptomycin (all Thermo Fisher Scientific, Inc.) at $37^{\circ} \mathrm{C}$ in a humidified incubator with $5 \% \mathrm{CO}_{2}$.

To knock down SIK2 or overexpress tripartite motif containing 28 (TRIM28), HCT116 cells were seeded into 6 -well plates at a density of $5 \times 10^{5}$ cells/well and transfected with $100 \mathrm{nM}$ Silencer Select small interfering RNA (siRNA)-SIK2 (cat. no. s23355) and Silencer Select Negative Control No. 1 siRNA (siRNA-NC; cat. no. 4390843; both from Thermo Fisher Scientific, Inc.) or $4 \mu \mathrm{g}$ pCDNA3.1-TRIM28 and pCDNA3.1-NC [Yunzhou Biotechnology (Guangzhou) Co., Ltd.], using Lipofectamine ${ }^{\circledR} 2000$ reagent (Invitrogen; Thermo Fisher Scientific, Inc.), according to the manufacturer's instructions, at $37^{\circ} \mathrm{C}$ for $5 \mathrm{~h}$. Then, the cells were incubated in DMEM supplemented with $10 \% \mathrm{FBS}$ at $37^{\circ} \mathrm{C}$ for $48 \mathrm{~h}$. Following incubation for $48 \mathrm{~h}$ and the evaluation of transfection efficacy, the cells were utilized for subsequent assays.

Reverse transcription-quantitative PCR (RT-qPCR). Total RNA was extracted from cells using the MolPure ${ }^{\circledR}$ cell RNA kit (Shanghai Yeasen Biotechnology Co., Ltd.) and cDNA was synthesized using a RT system kit (cat. no. N8080234; Invitrogen; Thermo Fisher Scientific, Inc.) according to the manufacturer's instructions. Subsequently, for qPCR, the QuantiNova SYBR Green PCR kit (Qiagen $\mathrm{GmbH}$ ) was used according to the manufacturer's instructions. The qPCR thermocycling conditions were as follows: Initial denaturation at $95^{\circ} \mathrm{C}$ for $10 \mathrm{sec}$, followed by 40 cycles of denaturation at $95^{\circ} \mathrm{C}$ for $10 \mathrm{sec}$, annealing at $60^{\circ} \mathrm{C}$ for $10 \mathrm{sec}$ and extension at $72^{\circ} \mathrm{C}$ for $15 \mathrm{sec}$. GAPDH served as the internal reference control. The differences in the expression of the target genes were assessed using the $2^{-\Delta \Delta \mathrm{Cq}}$ method (23). The primer sequences used are listed in Table I.

Western blot analysis. Total proteins were extracted from cells using a pre-chilled RIPA lysis buffer (Beyotime Institute of Biotechnology) for $10 \mathrm{~min}$ on ice and the protein concentration was determined using a BCA kit (cat. no. P0012; Beyotime Institute of Biotechnology). The protein samples $(25 \mu \mathrm{g} / \mathrm{lane})$ were separated via $12 \%$ SDS-PAGE. Subsequently, proteins were transferred onto PVDF membranes. Following blocking with $5 \%$ skimmed milk for $2 \mathrm{~h}$ at room temperature, the membranes were incubated with primary antibodies against SIK2 (cat. no. PA1-41208; dilution, 1:500; Invitrogen; Thermo Fisher Scientific, Inc.), MMP12 (cat. no. ab52897; dilution, 1:2,000), MMP9 (cat. no. ab76003; dilution, 1:1,000), glucose transporter 1 (Glut1; cat. no. ab115730; dilution, 1:50,000), hexokinase 2 (HK2; cat. no. ab209847; dilution, 1:1,000), pyruvate kinase (PK) M2 (PKM2; cat. no. ab85555; dilution, 1:1,000), TRIM28 (cat. no. ab109287; dilution, 1:10,000) and GAPDH (cat. no. ab8245; dilution, 1:1,000; all from Abcam) at $37^{\circ} \mathrm{C}$ overnight. The PVDF membranes were then incubated with the corresponding HRP goat anti-rabbit secondary antibody (cat. no. G-21234; dilution, 1: 50,000; Invitrogen; Thermo Fisher Scientific, Inc.) for $2 \mathrm{~h}$ at room temperature, followed by washing with PBS. The chemiluminescent reaction was performed using an ECL kit (iGene Biotechnology Co., Ltd.). GAPDH served as the internal reference and the results were analyzed using ImageJ software (v. 1.6; National Institutes of Health).

Cell Counting Kit-8 (CCK-8) assay. For CCK-8 assays, HCT116 cells were inoculated into a 96-well plate at a density of $5 \times 10^{3}$ cells/well. Following cell culture for $0,24,48$ and $72 \mathrm{~h}$ at $37^{\circ} \mathrm{C}$ in a $5 \% \mathrm{CO}_{2}$ incubator, each well was supplemented with $10 \mu \mathrm{l}$ CCK- 8 reagent (Beyotime Institute of Biotechnology), according to the manufacturer's instructions, and cells were incubated for an additional $1 \mathrm{~h}$ at $37^{\circ} \mathrm{C}$ in a $5 \% \mathrm{CO}_{2}$ incubator. The optical density was measured at a wavelength of $450 \mathrm{~nm}$ using a microplate reader (Molecular Devices, LLC).

Wound healing assay. HCT116 cells were seeded into a 6-well plate at a density of $5 \times 10^{5}$ cells/well until reaching $80 \%$ confluence. A linear scratch was created with a $200-\mu 1$ pipette tip in the middle of wells and free cells were removed by washing with PBS. Following culture in serum-free DMEM (Gibco; Thermo Fisher Scientific, Inc.) at $37^{\circ} \mathrm{C}$ for $24 \mathrm{~h}$, images of the wound area were captured in the same field at 0 and $24 \mathrm{~h}$ using a light microscope (Nikon Corporation; magnification, x100) and data were analyzed using ImageJ (v. 1.8.0; National Institutes of Health).

Transwell assay. Transwell inserts for $24-w e l l$ plates $(8 \mu \mathrm{m}$; Corning, Inc.) were coated with pre-diluted Matrigel (1:8; BD Biosciences) at $37^{\circ} \mathrm{C}$ for $30 \mathrm{~min}$. HCT116 cells, at a density of $5 \times 10^{5}$ cells in $200 \mu 1$ DMEM, were seeded into the Matrigel-coated upper chamber, while the lower chamber was filled with $400 \mu 1$ DMEM supplemented with 10\% FBS. Following incubation at $37^{\circ} \mathrm{C}$ for $24 \mathrm{~h}$, the cells on the lower surface of the membrane were fixed with $10 \%$ formaldehyde and stained with $0.1 \%$ crystal violet solution both for $15 \mathrm{~min}$ at room temperature. The number of invading cells was counted under a light microscope (Nikon Corporation; magnification, x100) and cells were analyzed using ImageJ (v. 1.8.0; National Institutes of Health). 
Table I. Primer sequences used for reverse transcription-quantitative PCR analysis

\begin{tabular}{|c|c|}
\hline Gene & Sequence \\
\hline SIK2 & $\begin{array}{l}\text { Forward: 5'-GGGTGGGGTTCTACGACATC-3' } \\
\text { Reverse: 5'-TATTGCCACCTCCGTCTTGG-3' }\end{array}$ \\
\hline Glut1 & $\begin{array}{l}\text { Forward: 5'-CCCCGTCCTGCTGCTATTG-3' } \\
\text { Reverse: 5'-GCACCGTGAAGATGATGAAGAC-3' }\end{array}$ \\
\hline HK2 & $\begin{array}{l}\text { Forward: 5'-CAAAGTGACAGTGGGTGTGG-3' } \\
\text { Reverse: 5'-GCCAGGTCCTTCACTGTCTC-3' }\end{array}$ \\
\hline PKM2 & $\begin{array}{l}\text { Forward:5'-CTTGCAATTATTTGAGGAACTCCGC-3' } \\
\text { Reverse: 5'-CACGGTACAGGTGGGCCTGAC-3' }\end{array}$ \\
\hline TRIM28 & $\begin{array}{l}\text { Forward: 5'-AGCGGGTGAAAAACACCAAG-3' } \\
\text { Reverse: 5'-ACCCAAAGCCATAGCCTTCC-3' }\end{array}$ \\
\hline GAPDH & $\begin{array}{l}\text { Forward: 5'-TCCACCACCCTGTTGCTGTA-3' } \\
\text { Reverse: 5'-ACCACAGTCCATGCCATCATC-3' }\end{array}$ \\
\hline
\end{tabular}

Glut1, glucose transporter 1; HK2, hexokinase 2; SIK2, salt-inducible kinase 2; PKM2, pyruvate kinase M2; TRIM28, tripartite motif containing 28 .

PK activity, and lactate and glycolysis detection. PK activity and lactate production in HCT116 cells were measured using a PK assay (cat. no. ab83432; Abcam) and a lactic acid assay (cat. no. BC2235; Beijing Solarbio Science \& Technology Co., Ltd.) kits, respectively. Glycolysis was measured using a glycolysis cell-based assay kit (cat. no. 600450-1; AmyJet Scientific, Inc.). The analyses were conducted according to the manufacturer's instructions.

Co-immunoprecipitation (co-IP) assay. For co-IP experiments, cells were collected and lysed with pre-cooled IP lysis buffer (cat. no. P0013) containing protease inhibitors (cat. no. P1046; both from Beyotime Institute of Biotechnology) for $10 \mathrm{~min}$ on ice. The supernatant was collected after centrifugation at $13,000 \mathrm{x} \mathrm{g}$ for $10 \mathrm{~min}$ at $4{ }^{\circ} \mathrm{C}$. Subsequently, the lysate $(0.5 \mathrm{mg}$ per IP reaction) was supplemented with $1 \mu \mathrm{g}$ SIK (cat. no. 6919; dilution, 1:50; Cell Signaling Technology, Inc.) or TRIM28 antibody (cat. no. ab109545; dilution, 1:30; Abcam) and $10 \mu 1$ protein A agarose beads (Beyotime Institute of Biotechnology) followed by gentle rotation at $4^{\circ} \mathrm{C}$ for $4 \mathrm{~h}$. Following centrifugation at $1,000 \mathrm{x} \mathrm{g}$ for $3 \mathrm{~min}$ at $4^{\circ} \mathrm{C}$, the supernatant was carefully removed and the agarose beads in the bottom of the tube were washed with lysis buffer. The pellets were then dissolved in $15 \mu 12 \mathrm{X}$ SDS sample buffer and boiled for $5 \mathrm{~min}$, followed by western blot analysis.

Bioinformatics analyses. The Broad Institute Cancer Cell Line Encyclopedia (CCLE; https://portals.broadinstitute. org/ccle) (24) incorporates genetic data from numerous human cancer cell lines and has become one of the standard databases for cancer genomics. Therefore, the mRNA expression levels of SIK2 in CRC were downloaded from the CCLE database. The Biological General Repository for Interaction Datasets (BioGRID; https://thebiogrid.org) (25) includes data on protein, genetic and drug interactions. In the present study, BioGRID was used to reveal the interactions between
SIK2 and other proteins, referring to https://thebiogrid. org/116840/table/homo-sapiens/sik2.html.

Statistical analysis. All experiments were repeated at least three times. Data are expressed as the mean \pm SD of triplicate experiments. All statistical analyses were carried out using GraphPad Prism 8.0 (GraphPad Software, Inc.). The differences between two groups were compared using an unpaired Student's t-test, while those among multiple groups with one-way ANOVA followed by Tukey's post hoc test. $\mathrm{P}<0.05$ was considered to indicate a statistically significant difference.

\section{Results}

Expression levels of SIK2 in CRC cell lines. The mRNA expression levels of SIK2 were significantly increased in all types of cancer in the CCLE database, including CRC (Fig. 1A). The mRNA and protein expression levels of SIK2 were determined in the normal colonic epithelial cell line, NCM460, and different CRC cell lines (LoVo, SW480, SW620, CW2 and HCT116) using RT-qPCR and western blot analysis, respectively. The results demonstrated that SIK2 was upregulated in all examined CRC cell lines compared with NCM460 cells (Fig. 1B and C). The expression of SIK2 was more potent in the HCT116 cell line; therefore, HCT116 cells were used for the subsequent experiments.

SIK2 silencing attenuates the proliferation, migration and invasion of CRC cells. The silencing potency of siRNA-SIK2 on the expression of SIK2 in HCT116 cells was evaluated using RT-qPCR and western blot analysis. It was found that siRNA-SIK2-1 exerted the strongest silencing effect on the expression of SIK2 compared with siRNA-SIK2-2, and it was therefore used in the knockdown experiments (Fig. 2A and B). Subsequently, HCT116 cells were divided into the following three groups: Control, siRNA-NC and siRNA-SIK2 groups. The proliferative ability of HCT116 cells was determined using 
A

mRNA expression (RNAseq): SIK2
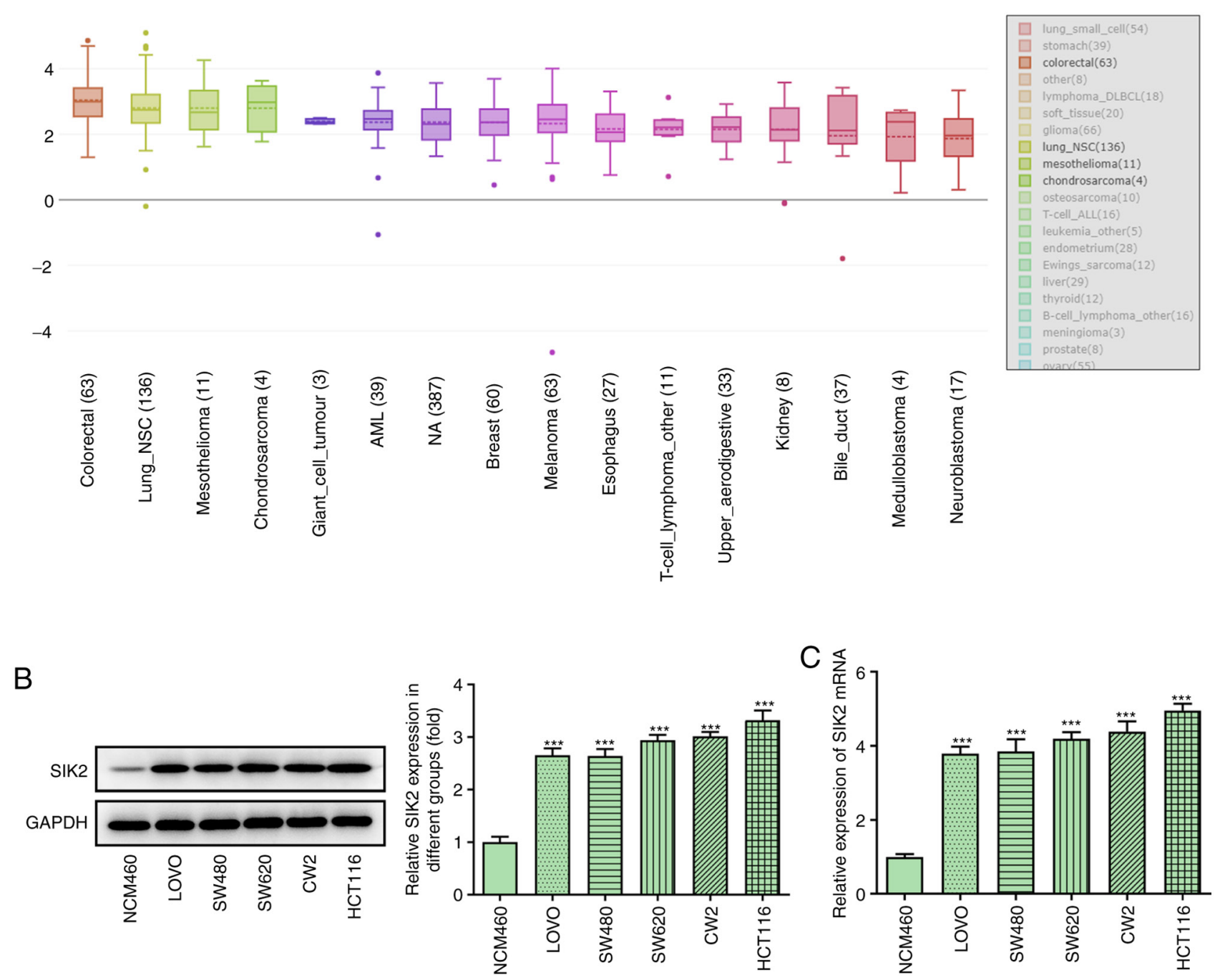

Figure 1. Expression level of SIK2 in CRC lines. (A) mRNA expression levels of SIK2 were notably increased in all types of cancer in the Cancer Cell Line Encyclopedia. The expression levels of SIK2 in the normal colonic epithelial cell line, NCM460, and different CRC cell lines (LoVo, SW480, SW620, CW2 and HCT116) were determined via (B) western blotting and (C) reverse transcription-quantitative PCR. ${ }^{* * *} \mathrm{P}<0.001$ vs. NCM460. CRC, colorectal cancer; SIK2, salt-inducible kinase 2 .

a CCK-8 assay. As shown in Fig. 2C, the cell proliferation rate in the siRNA-SIK2 group was notably lower compared with the siRNA-NC group at $24 \mathrm{~h}$ following cell transfection. This trend was maintained over time, suggesting that SIK2 knockdown could attenuate HCT116 cell proliferation (Fig. 2C). In addition, the cell migratory and invasive rates were measured in the same experimental groups. The wound healing assays revealed that the wound was almost completely closed in the siRNA-NC group at $24 \mathrm{~h}$ post-wounding. However, the migratory ability of HCT116 cells in the siRNA-SIK 2 group was significantly decreased (Fig. 2D). The Transwell assays also demonstrated that the invasive ability of HCT116 cells was decreased in the siRNA-SIK2 group (Fig. 2E). Furthermore, the protein expression levels of MMP12 and MMP9, which are relevant to cell movement (26), were measured via western blot analysis. Consistent with the Transwell assay results, both protein expression levels were downregulated in the siRNA-SIK2 group compared with the siRNA-NC group (Fig. 2F).
SIK2 knockdown attenuates glycolysis in CRC cell lines. The activity of PK, lactate production and glycolysis were evaluated using their corresponding kits. The levels of all the aforementioned processes were significantly attenuated following cell transfection with siRNA-SIK2 (Fig. 3A and B). Additionally, the expression levels of the glycolysis-related genes Glut1, HK2 and PKM2 were determined via RT-qPCR and western blot analyses. The results demonstrated that the expression levels of Glut1, HK2 and PKM2 were significantly downregulated in HCT116 cells transfected with siRNA-SIK2 compared with the siRNA-NC group (Fig. 3C and D).

Association between SIK2 and TRIM28. Bioinformatics analysis using the BioGRID database predicted that TRIM28 could interact with SIK2. Therefore, the expression levels of TRIM28 were determined in NCM460, LoVo, SW480, SW620, CW2 and HCT116 cells via RT-qPCR and western blot analysis. The results indicated that TRIM28 was upregulated in CRC cell lines (Fig. 4A and B). In addition, co-IP was conducted to verify 

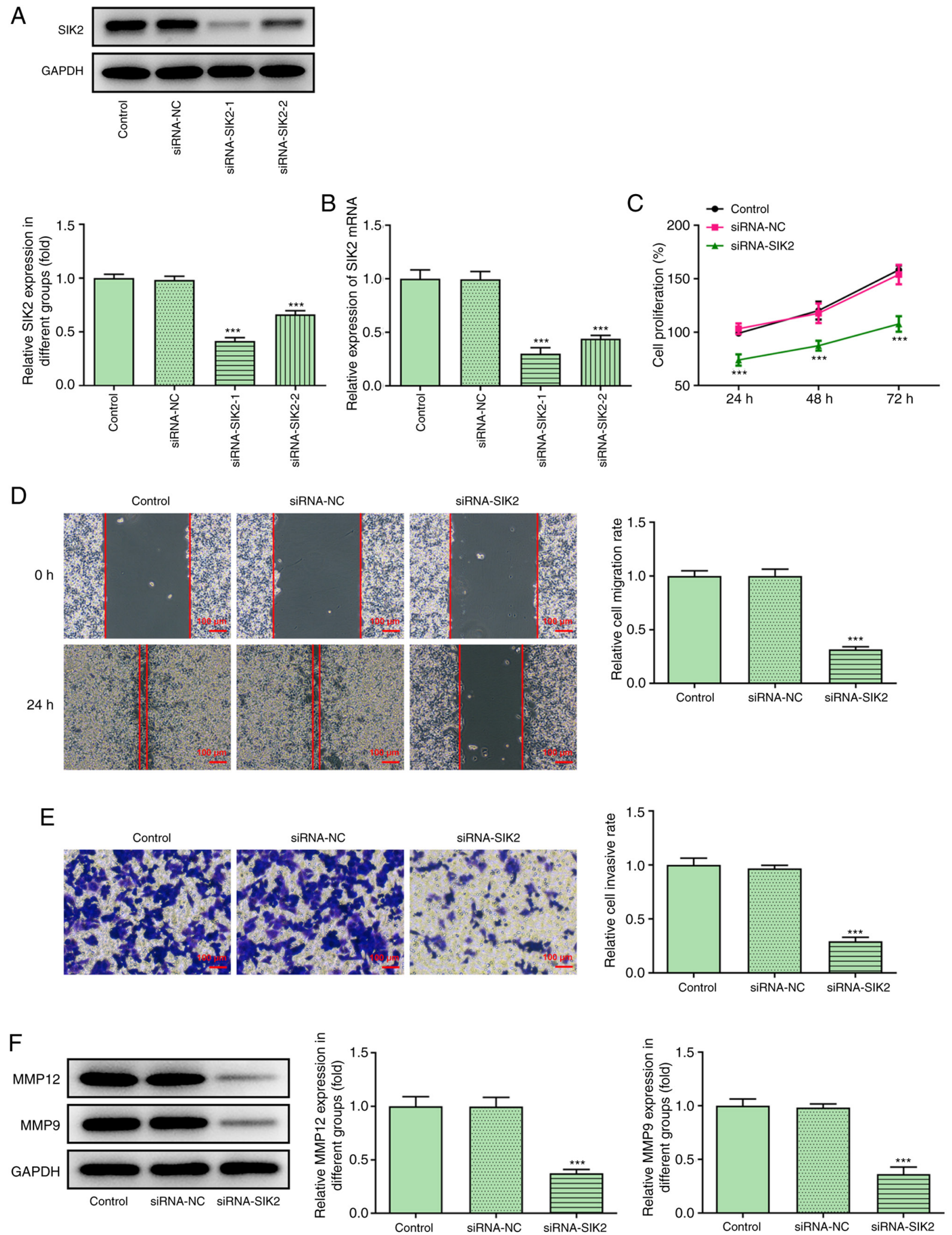

Figure 2. SIK2 silencing attenuates the proliferation, migration and invasion of colorectal cancer cells. The transfection efficiency in HCT116 cells transfected with siRNA-SIK2 was evaluated via (A) western blot and (B) reverse transcription-quantitative PCR analyses. (C) A Cell Counting Kit-8 assay was used to determine the proliferative ability of HCT116 cells. Cell migration and invasion rates were measured using (D) wound healing and (E) Transwell assays, respectively. Scale bar, $100 \mu \mathrm{m}$. (F) MMP12 and MMP9 protein expression levels were detected using western blot analysis. ${ }^{* * *} \mathrm{P}<0.001$ vs. siRNA-NC group. SIK2, salt-inducible kinase 2; siRNA, small interfering RNA; NC, negative control.

the interaction between SIK2 and TRIM28, and the results revealed that endogenous SIK2 was co-precipitated with endogenous TRIM28 in HCT116 cells (Fig. 4C and D). Furthermore,
TRIM28 mRNA and protein expression was downregulated in the siRNA-SIK2 group (Fig. 4E and F), supporting the interaction between SIK2 and TRIM28. 
A
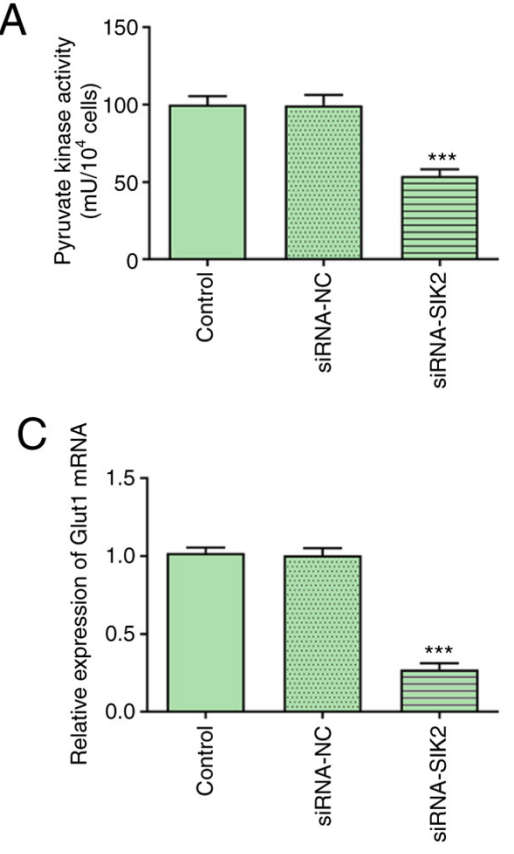
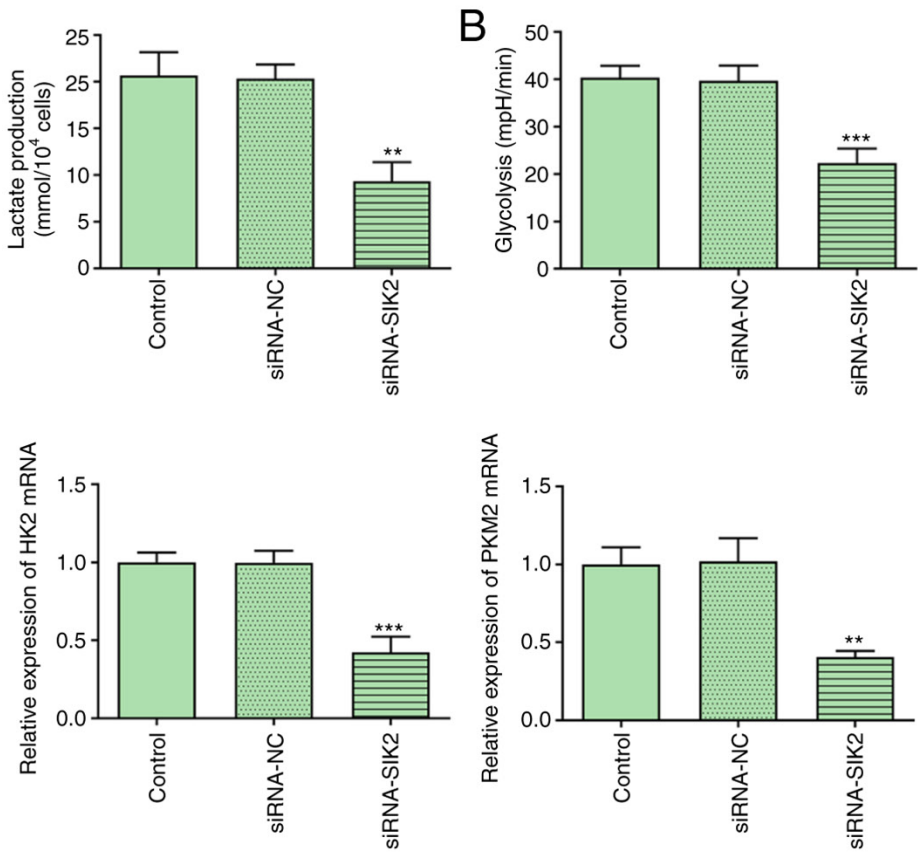
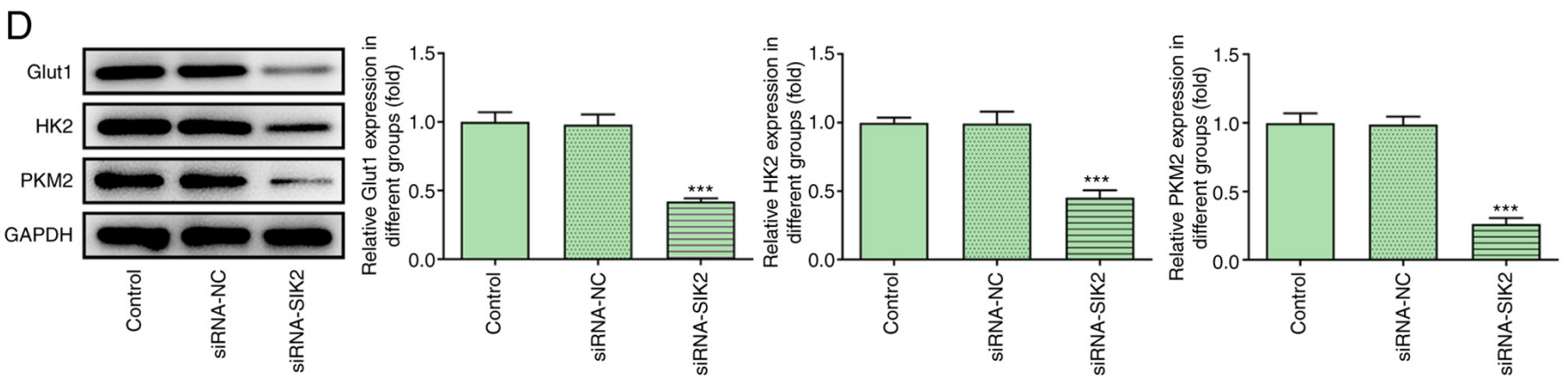

Figure 3. SIK2 silencing attenuates glycolysis in colorectal cancer cell lines. The levels of (A) pyruvate kinase activity, lactate production and (B) glycolysis were detected using their corresponding kits. The mRNA and protein expression levels of the glycolysis-related genes, Glut1, HK2 and PKM2, were determined via (C) reverse transcription-quantitative PCR and (D) western blot analyses, respectively. ${ }^{* * *} \mathrm{P}<0.01,{ }^{* * * *} \mathrm{P}<0.001$ vs. siRNA-NC group. PKM2, pyruvate kinase M2; Glut1, glucose transporter 1; HK2, hexokinase 2; siRNA, small interfering RNA; NC, negative control.

SIK2 regulates the proliferation, migration and invasion of CRC cells by regulating TRIM28. The transfection efficiency of HCT116 cells with pCDNA3.1-TRIM28 was assessed via RT-qPCR and western blot analyses, and the results demonstrated that TRIM28 was successfully overexpressed (Fig. 5A and B). Subsequently, HCT116 cells were divided into the following four groups: Control, siRNA-SIK2, siRNA-SIK2 + overexpression vector (ov)-NC and siRNA-SIK2 + ov-TRIM28 groups. The cell proliferation rate in the four different groups was measured using a CCK-8 assay. It was found that TRIM28 overexpression reversed the SIK2-silencing mediated decreased in the proliferative ability of HCT116 cells (Fig. 5C). Consistent with the CCK-8 assay results, the cell migration and invasion assays demonstrated that the reduced migratory and invasive abilities of HCT116 cells with SIK2 knockdown were restored following TRIM28 overexpression (Fig. 5D and E). Furthermore, MMP12 and MMP9 expression was upregulated in the siRNA-SIK $2+$ ov-TRIM28 group compared with the siRNA-SIK $2+$ ov-NC group (Fig. 5F). Collectively, the aforementioned findings indicated that SIK 2 could promote the proliferation, migration and invasion of HCT116 cells by upregulating TRIM28.
SIK2 modulates glycolysis in CRC cell lines by regulating TRIM28. The effect of TRIM28 on glycolysis in HCT116 cells was then evaluated following TRIM28 overexpression. The levels of PK activity, lactate secretion and glycolysis were all restored in the siRNA-SIK2 + ov-TRIM28 group compared with the siRNA-SIK2 + ov-NC group (Fig. 6A and B). Similarly, the RT-qPCR and western blotting results demonstrated that the expression levels of Glut1,HK2 and PKM2 were reversed following TRIM28 overexpression (Fig. 6C and D). Collectively, these results suggested that SIK 2 could promote glycolysis in HCT116 cells by upregulating TRIM28.

\section{Discussion}

CRC is a common malignant tumor, and its incidence in the elderly population is decreasing with the wider application of colonoscopy in the clinical practice. However, the morbidity and mortality rates of CRC in young individuals are increasing, particularly in developing countries (27). In addition, due to the lack of typical clinical symptoms in the early stages of the disease, numerous patients with CRC are already at an advanced stage at the time of diagnosis. Therefore, the 
A

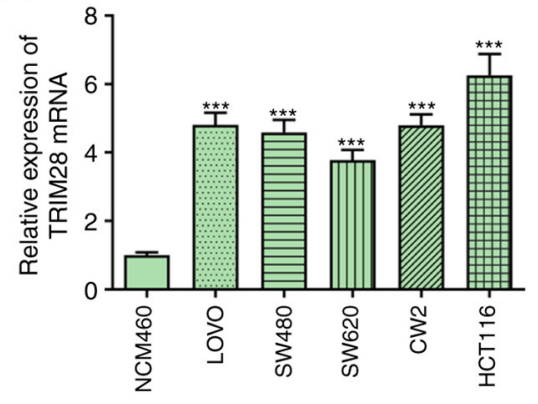

C

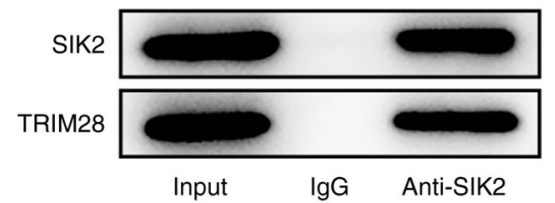

B

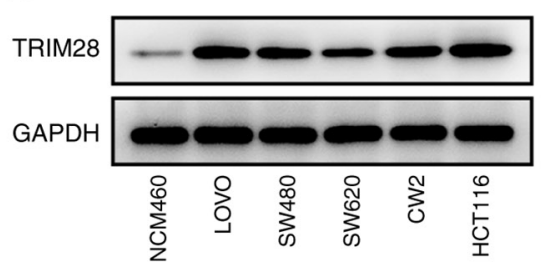

$\mathrm{D}$
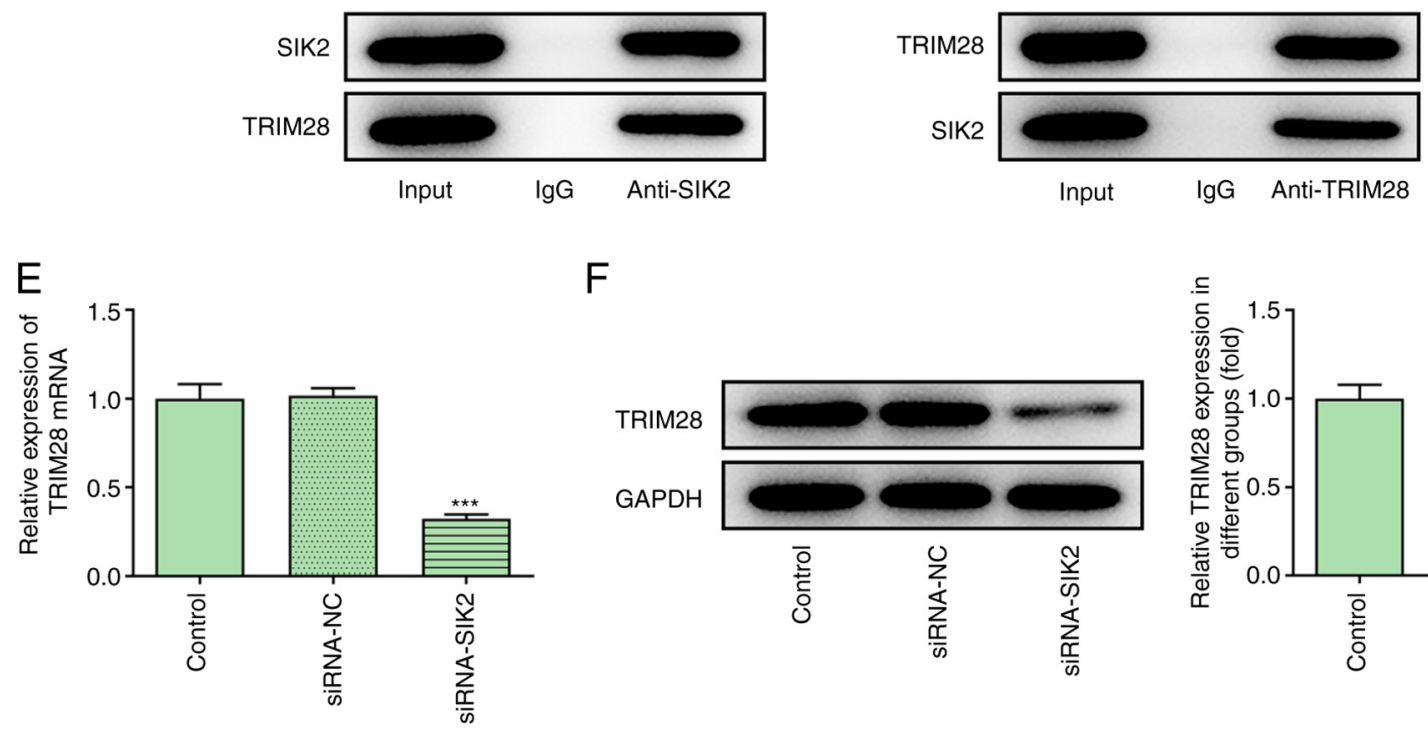

$\mathrm{F}$
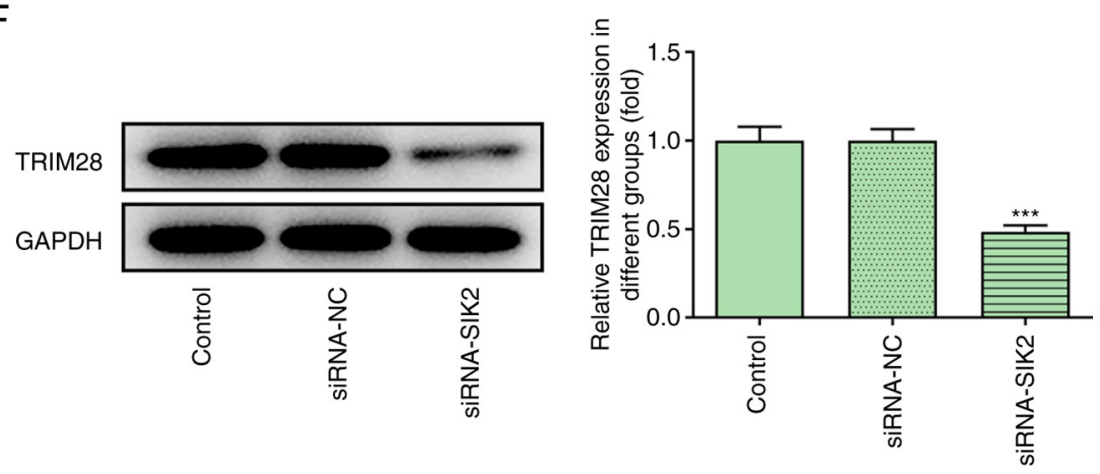

Figure 4. Association between SIK2 and TRIM28. The mRNA and protein expression levels of TRIM28 in NCM460, LoVo, SW480, SW620, CW2 and HCT116 cells were measured using (A) reverse transcription-quantitative PCR and (B) western blot analyses, respectively. ${ }^{* * *} \mathrm{P}<0.001 \mathrm{vs}$. NCM460. (C and D) Co-immunoprecipitation assays were conducted to verify the association between SIK2 and TRIM28. TRIM28 (E) mRNA and (F) protein expression levels in HCT116 cells. ${ }^{* * *} \mathrm{P}<0.001$ vs. siRNA-NC group. SIK2, RNA salt-inducible kinase 2; TRIM28, tripartite motif containing 28; siRNA, small interfering RNA; NC, negative control.

prognosis for these patients is usually poor (28). In a study on the association between CRC and geographic location and diet, Doll and Peto (29) revealed that $>90 \%$ of intestinal tumors were associated with dietary habits, suggesting that different foods could have a great influence on the composition and diversity of the gut microbiome (30). Moreover, Liang et al (31) showed that the structure of the intestinal microbial community of patients with colorectal adenoma was more susceptible to interference compared with that of healthy subjects. Since the progression from detectable precancerous lesions to $\mathrm{CRC}$ is relatively slow, patients diagnosed early usually have a good prognosis and, therefore, CRC screening has been shown to be effective in reducing mortality (32). Therefore, early diagnosis is considered particularly important for disease outcome.

Cancer cells undergo a series of changes during the process of carcinogenic transformation and the changes in cell metabolism serve a key role for their new phenotypes (33). Compared with normal cells, cancer cells exhibit enhanced proliferation and can rapidly adapt to changes in metabolism, thereby meeting the requirements of cells for metabolism and biosynthesis $(34,35)$. The most significant feature of metabolism is the ability of cancer cells to increase the glycolysis rate in an aerobic environment, which is referred to as aerobic glycolysis (36). Glycolysis is the degradation reaction of glucose or glycogen into lactic acid or pyruvate that resembles fermentation, thus resulting in reduced energy consumption and ATP production from the cells (37). The glycolytic enzymes PKM2 and HK2 and the glucose transporter Glut1 are often dysregulated during carcinogenesis, and they have been considered to be involved in the crucial pathways of aerobic glycolysis in cancer cells (38). Previous studies reported that SIK2 was involved in the promotion of aerobic glycolysis in breast and ovarian cancer cells $(20,39)$. In addition to studying the effect of SIK2 on the proliferation, migration and invasion of CRC cells, the present study aimed to investigate the effect of SIK2 on glycolysis. The results indicated that SIK2 could also promote glycolysis in CRC cells, thus suggesting that SIK2 may be a promising biomarker for CRC.

The current study also investigated the specific mechanism underlying the effects of SIK2 on CRC. Bioinformatics analysis using the BioGRID database revealed that TRIM28 could be a target of SIK2. The association between the 
A

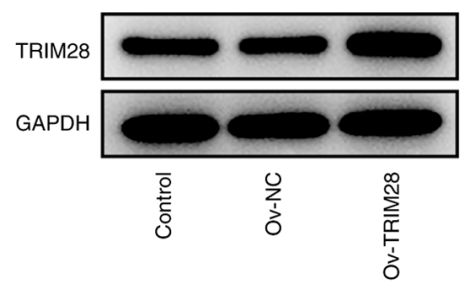

B

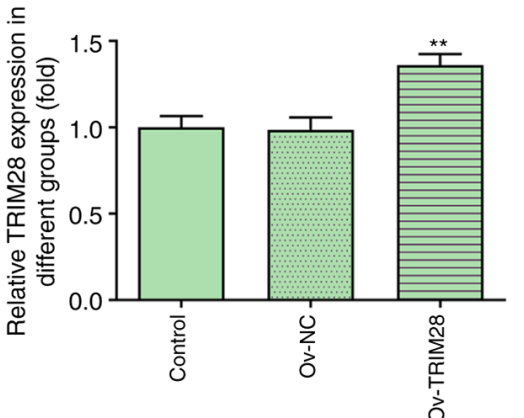

D Control $\quad$ siRNA-SIK2

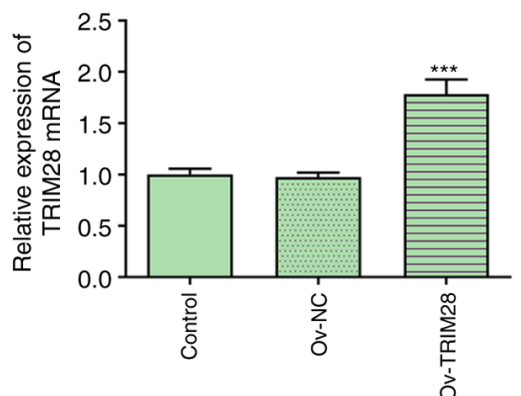

SiRNA-SIK2+Ov-NC SiRNA-SIK2+Ov-TRIM28

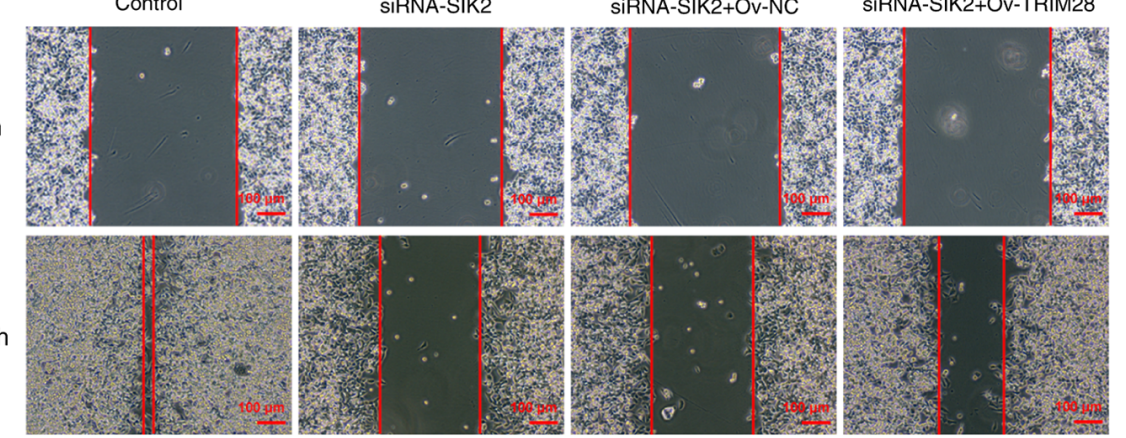

$\mathrm{oh}$

$E$

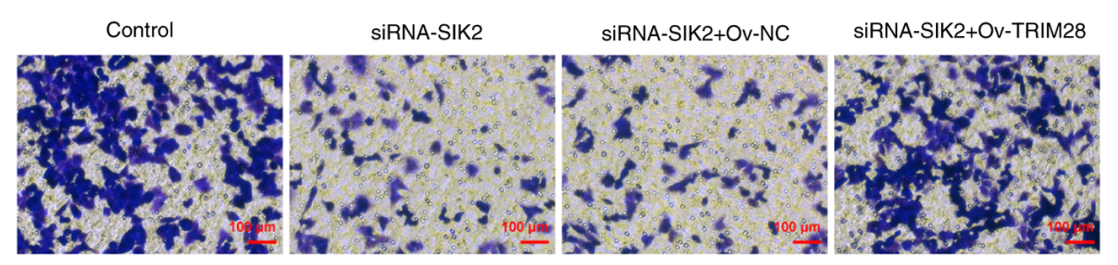

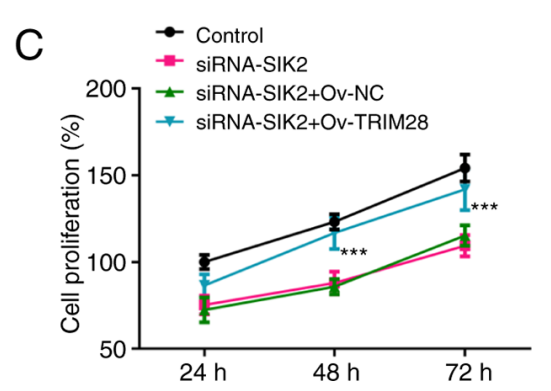
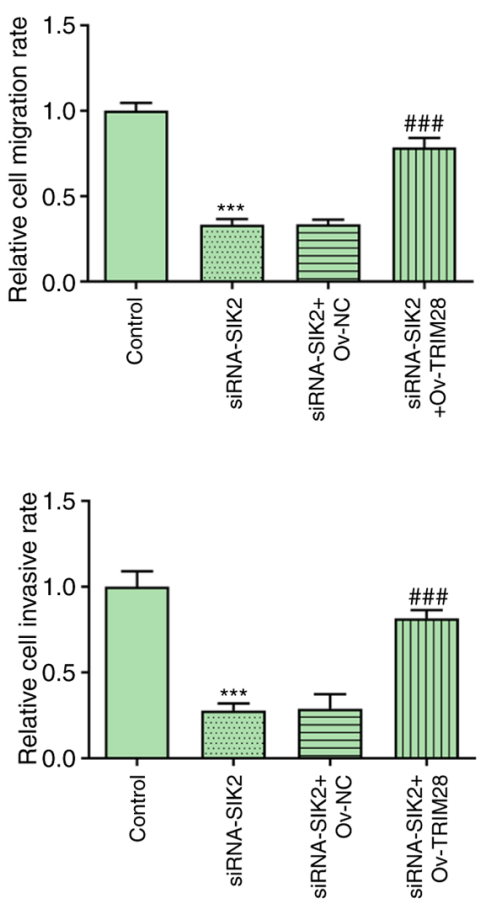
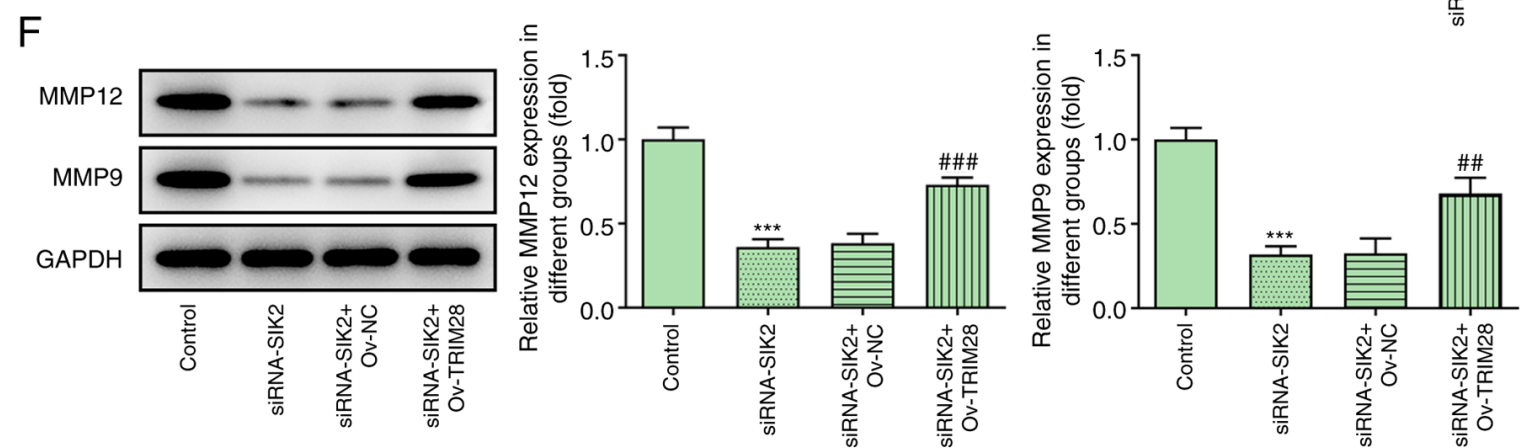

Figure 5. SIK2 regulates the proliferation, migration and invasion of colorectal cancer cell lines by regulating TRIM28. The transfection efficiency of cells with pCDNA3.1-TRIM28 was confirmed via (A) western blot and (B) reverse transcription-quantitative PCR analyses. ${ }^{*} \mathrm{P}<0.01,{ }^{* * * *} \mathrm{P}<0.001$ vs. ov-NC group. (C) Cell proliferation ability in different groups was evaluated using a Cell Counting Kit-8 assay. ${ }^{* * *} \mathrm{P}<0.001$ vs. siRNA-SIK2 + ov-NC group. Cell migration and invasion were measured using (D) wound healing and (E) Transwell assays, respectively. Scale bar, $100 \mu \mathrm{m}$. (F) Expression levels of MMP12 and MMP9 were determined via western blot analysis. ${ }^{* * *} \mathrm{P}<0.001$ vs. control group; ${ }^{* \#} \mathrm{P}<0.01,{ }^{\# \#} \mathrm{P}<0.001$ vs. siRNA-SIK2 + ov-NC group. SIK2, salt-inducible kinase 2; TRIM28, tripartite motif containing 28; ov, overexpression vector; NC, negative control; siRNA, small interfering RNA.

two proteins was verified using a co-IP assay. It has been reported that TRIM28 is involved in several types of cancer. For example, increased expression of TRIM28 was found to be associated with cervical cancer metastasis (40). In gastric cancer, TRIM28 was upregulated in the peripheral blood of patients and its expression was associated with 

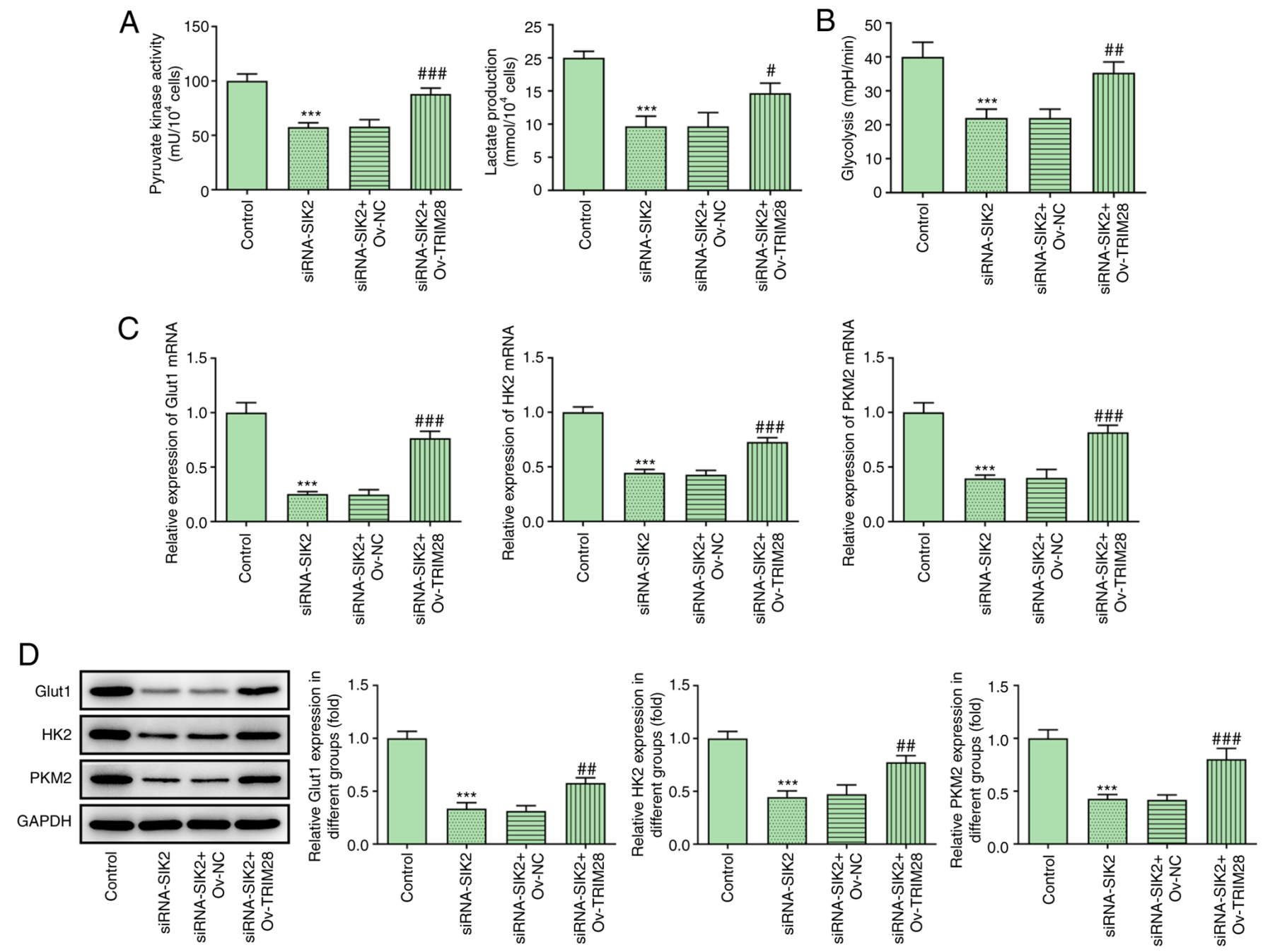

Figure 6. SIK2 regulates glycolysis in colorectal cancer cell lines by regulating TRIM28. The levels of (A) pyruvate kinase activity, lactate production and (B) glycolysis were measured using their corresponding kits. The mRNA and protein expression levels of the glycolysis-related genes Glut1, HK2 and PKM2 were determined via (C) reverse transcription-quantitative PCR and (D) western blot analyses, respectively. ${ }^{* * *} \mathrm{P}<0.001$ vs. control group; ${ }^{*} \mathrm{P}<0.05$, ${ }^{\# \#} \mathrm{P}<0.01$ and ${ }^{\# \# \#} \mathrm{P}<0.001$ vs. siRNA-SIK2 + Ov-NC group. SIK2, salt-inducible kinase 2; PKM2, pyruvate kinase M2; TRIM28, tripartite motif containing 28; ov, overexpression vector; NC, negative control; siRNA, small interfering RNA; Glut1, glucose transporter 1; HK2, hexokinase 2.

poor prognosis $(41,42)$. In gliomas, TRIM28 was also highly expressed and was positively associated with the degree of tumor malignancy, poor overall survival and progression-free survival (43). Interestingly, the expression of TRIM28 was elevated in the interstitial tissues of patients with CRC, while its increased expression was associated with worse prognosis $(44,45)$. In the present study, TRIM28 overexpression reversed the effects of SIK2 silencing on cell proliferation, migration, invasion and glycolysis, thus indicating that the effects of SIK2 on the physiological behavior of CRC cells may be mediated by regulating TRIM28. However, as only in vitro experiments were performed, the results of the present study must be further verified using in vivo experiments in the future. Moreover, the mechanism underlying the downstream genes of TRIM 28 are worthy of a follow-up investigation.

In conclusion, the results of the present study demonstrated that SIK2 was upregulated in CRC, while SIK2 knockdown suppressed cell proliferation, migration, invasion and glycolysis, suggesting that SIK2 may represent a promising biomarker for CRC. Furthermore, TRIM28 overexpression could reverse these effects, thus indicating that SIK2 may affect the malignant behavior and glycolysis of CRC cells by regulating TRIM28. The findings of the present study may provide a novel approach to the targeted therapy and clinical diagnosis of $\mathrm{CRC}$ in the future.

\section{Acknowledgements}

Not applicable.

\section{Funding}

No funding was received.

\section{Availability of data and materials}

The datasets generated and/or analyzed during the current study are available from the corresponding author upon reasonable request. 


\section{Authors' contributions}

$\mathrm{XN}$ designed and performed the experiments and made substantial contributions to the manuscript writing. YF performed the experiments and analyzed the data. XF made substantial contributions to conception and design, guided the experiments and revised the manuscript. All the authors have read and approved the final manuscript. XN and XF confirm the authenticity of the raw data.

\section{Ethics approval and consent to participate}

Not applicable.

\section{Patient consent for publication}

Not applicable.

\section{Competing interests}

The authors declare that they have no competing interests.

\section{References}

1. Janney A, Powrie F and Mann EH: Host-microbiota maladaptation in colorectal cancer. Nature 585: 509-517, 2020.

2. Bray F, Ferlay J, Soerjomataram I, Siegel RL, Torre LA and Jemal A: Global cancer statistics 2018: GLOBOCAN estimates of incidence and mortality worldwide for 36 cancers in 185 countries. CA Cancer J Clin 68: 394-424, 2018.

3. Johdi NA and Sukor NF: Colorectal cancer immunotherapy: Options and strategies. Front Immunol 11: 1624, 2020.

4. Schmoll HJ, Van Cutsem E, Stein A, Valentini V, Glimelius B, Haustermans K, Nordlinger B, van de Velde CJ, Balmana J, Regula J, et al: ESMO consensus guidelines for management of patients with colon and rectal cancer. A personalized approach to clinical decision making. Ann Oncol 23: 2479-2516, 2012.

5. Van Cutsem E, Cervantes A, Nordlinger B and Arnold D; ESMO Guidelines Working Group: Metastatic colorectal cancer: ESMO clinical practice guidelines for diagnosis, treatment and follow-up. Ann Oncol 25 (Suppl 3): iiil-iii9, 2014.

6. Yoshino T, Arnold D, Taniguchi H, Pentheroudakis G, Yamazaki K, Xu RH, Kim TW, Ismail F, Tan IB, Yeh KH, et al Pan-Asian adapted ESMO consensus guidelines for the management of patients with metastatic colorectal cancer: A JSMO-ESMO initiative endorsed by CSCO, KACO, MOS, SSO and TOS. Ann Oncol 29: 44-70, 2018.

7. Van Cutsem E, Cervantes A, Adam R, Sobrero A, Van Krieken JH, Aderka D, Aranda Aguilar E, Bardelli A, Benson A, Bodoky G, et al: ESMO consensus guidelines for the management of patients with metastatic colorectal cancer. Ann Oncol 27: 1386-1422, 2016

8. Bonjer HJ, Deijen CL, Abis GA, Cuesta MA, van der Pas MH de Lange-de Klerk ES, Lacy AM, Bemelman WA, Andersson J, Angenete E, et al: A randomized trial of laparoscopic versus open surgery for rectal cancer. N Engl J Med 372: 1324-1332, 2015.

9. MacFarlane JK, Ryall RD and Heald RJ: Mesorectal excision for rectal cancer. Lancet 341: 457-460, 1993.

10. Park SC, Sohn DK, Kim MJ, Chang HJ, Han KS, Hyun JH, Joo J and $\mathrm{Oh} \mathrm{JH}$ : Phase II clinical trial to evaluate the efficacy of transanal endoscopic total mesorectal excision for rectal cancer. Dis Colon Rectum 61: 554-560, 2018.

11. Miller KD, Nogueira L, Mariotto AB, Rowland JH, Yabroff KR, Alfano CM, Jemal A, Kramer JL and Siegel RL: Cancer treatment and survivorship statistics, 2019. CA Cancer J Clin 69: 363-385, 2019.

12. Garg MB, Lincz LF, Adler K, Scorgie FE, Ackland SP and Sakoff JA: Predicting 5-fluorouracil toxicity in colorectal cancer patients from peripheral blood cell telomere length: A multivariate analysis. Br J Cancer 107: 1525-1533, 2012.
13. Ogura A, Konishi T, Cunningham C, Garcia-Aguilar J, Iversen $\mathrm{H}$, Toda S, Lee IK, Lee HX, Uehara K, Lee P, et al: Neoadjuvant (chemo) radiotherapy with total mesorectal excision only is not sufficient to prevent lateral local recurrence in enlarged nodes: Results of the multicenter lateral node study of patients with low cT3/4 rectal cancer. J Clin Oncol 37: 33-43, 2019.

14. Arnold M, Sierra MS, Laversanne M, Soerjomataram I, Jemal A and Bray F: Global patterns and trends in colorectal cancer incidence and mortality. Gut 66: 683-691, 2017.

15. Patel JN: Application of genotype-guided cancer therapy in solid tumors. Pharmacogenomics 15: 79-93, 2014.

16. Pellino G, Gallo G, Pallante P, Capasso R, De Stefano A, Maretto I, Malapelle U, Qiu S, Nikolaou S, Barina A, et al: Noninvasive biomarkers of colorectal cancer: Role in diagnosis and personalised treatment perspectives. Gastroenterol 2018: 2397863, 2018

17. Ogunwobi OO, Mahmood F and Akingboye A: Biomarkers in colorectal cancer: Current research and future prospects. Int J Mol Sci 21: 5311, 2020.

18. Patel JN, Fong MK and Jagosky M: Colorectal cancer biomarkers in the era of personalized medicine. J Pers Med 9: 3, 2019.

19. Yiu AJ and Yiu CY: Biomarkers in colorectal cancer. Anticancer Res 36: 1093-1102, 2016.

20. Zong S, Dai W, Fang W, Guo X and Wang K: SIK2 promotes cisplatin resistance induced by aerobic glycolysis in breast cancer cells through PI3K/AKT/mTOR signaling pathway. Biosci Rep: May 27, 2020 (Epub Ahead of Print).

21. Zhao J, Zhang X, Gao T, Wang S, Hou Y, Yuan P, Yang Y, Yang T, Xing J, Li J and Liu S: SIK2 enhances synthesis of fatty acid and cholesterol in ovarian cancer cells and tumor growth through PI3K/Akt signaling pathway. Cell Death Dis 11: 25 , 2020.

22. Sun Z, Niu S, Xu F, Zhao W, Ma R and Chen M: CircAMOTL1 promotes tumorigenesis through miR-526b/SIK2 axis in cervical cancer. Front Cell Dev Biol 8: 568190, 2020.

23. Livak KJ and Schmittgen TD: Analysis of relative gene expression data using real-time quantitative PCR and the 2(-Delta Delta C (T)) method. Methods 25: 402-408, 2001.

24. Barretina J, Caponigro G, Stransky N, Venkatesan K, Margolin AA, Kim S, Wilson CJ, Lehár J, Kryukov GV, Sonkin D, et al: The cancer cell line encyclopedia enables predictive modelling of anticancer drug sensitivity. Nature 483: 603-607, 2012.

25. Oughtred R, Rust J, Chang C, Breitkreutz BJ, Stark C, Willems A, Boucher L, Leung G, Kolas N, Zhang F, et al: The BioGRID database: A comprehensive biomedical resource of curated protein, genetic, and chemical interactions. Protein Sci 30: $187-200,2021$.

26. Li J, Xu X, Jiang Y, Hansbro NG, Hansbro PM, Xu J and Liu G: Elastin is a key factor of tumor development in colorectal cancer. BMC Cancer 20: 217, 2020.

27. Mauri G, Sartore-Bianchi A, Russo AG, Marsoni S, Bardelli A and Siena S: Early-onset colorectal cancer in young individuals. Mol Oncol 13: 109-131, 2019.

28. Zhang X, Zhang H, Shen B and Sun XF: Chromogranin-A expression as a novel biomarker for early diagnosis of colon cancer patients. Int J Mol Sci 20: 2919, 2019.

29. Doll R and Peto R: The causes of cancer: Quantitative estimates of avoidable risks of cancer in the United States today. J Natl Cancer Inst 66: 1191-1308, 1981.

30. Rothschild D, Weissbrod O, Barkan E, Kurilshikov A, Korem T, Zeevi D, Costea PI, Godneva A, Kalka IN, Bar N, et al: Environment dominates over host genetics in shaping human gut microbiota. Nature 555: 210-215, 2018.

31. Liang Q, Chiu J, Chen Y, Huang Y, Higashimori A, Fang J, Brim H, Ashktorab H, Ng SC, Ng SSM, et al: Fecal bacteria act as novel biomarkers for noninvasive diagnosis of colorectal cancer. Clin Cancer Res 23: 2061-2070, 2017.

32. Ladabaum U, Dominitz JA, Kahi C and Schoen RE: Strategies for colorectal cancer screening. Gastroenterology 158: 418-432, 2020.

33. Hanahan D and Weinberg RA: Hallmarks of cancer: The next generation. Cell 144: 646-674, 2011.

34. Cuezva JM, Ortega AD, Willers I, Sánchez-Cenizo L, Aldea M and Sánchez-Aragó M: The tumor suppressor function of mitochondria: Translation into the clinics. Biochim Biophys Acta 1792: 1145-1158, 2009. 
35. Vander Heiden MG, Lunt SY, Dayton TL, Fiske BP, Israelsen WJ, Mattaini KR, Vokes NI, Stephanopoulos G, Cantley LC, Metallo CM and Locasale JW: Metabolic pathway alterations that support cell proliferation. Cold Spring Harb Symp Quant Biol 76: 325-334, 2011.

36. Lunt SY and Vander Heiden MG: Aerobic glycolysis: Meeting the metabolic requirements of cell proliferation. Annu Rev Cell Dev Biol 27: 441-464, 2011.

37. Enzo E, Santinon G, Pocaterra A, Aragona M, Bresolin S, Forcato M, Grifoni D, Pession A, Zanconato F, Guzzo G, et al: Aerobic glycolysis tunes YAP/TAZ transcriptional activity. EMBO J 34: 1349-1370, 2015.

38. Samec M, Liskova A, Koklesova L, Samuel SM, Zhai K, Buhrmann C, Varghese E, Abotaleb M, Qaradakhi T, Zulli A, et al: Flavonoids against the warburg phenotype-concepts of predictive, preventive and personalised medicine to cut the Gordian knot of cancer cell metabolism. EPMA J 11: 377-398, 2020.

39. Gao T, Zhang X, Zhao J, Zhou F, Wang Y, Zhao Z, Xing J, Chen B, Li J and Liu S: SIK2 promotes reprogramming of glucose metabolism through PI3K/AKT/HIF-1 $\alpha$ pathway and Drp1-mediated mitochondrial fission in ovarian cancer. Cancer Letters 469: 89-101, 2020.

40. Lin LF, Li CF, Wang WJ, Yang WM, Wang DD, Chang WC, Lee WH and Wang JM: Loss of ZBRK1 contributes to the increase of KAP1 and promotes KAP1-mediated metastasis and invasion in cervical cancer. PLoS One 8: e73033, 2013.
41. Yokoe T, Toiyama Y, Okugawa Y, Tanaka K, Ohi M, Inoue Y, Mohri Y, Miki C and Kusunoki M: KAP1 is associated with peritoneal carcinomatosis in gastric cancer. Ann Surg Oncol 17: 821-828, 2010

42. Wang YY, Li L, Zhao ZS and Wang HJ: Clinical utility of measuring expression levels of KAP1, TIMP1 and STC2 in peripheral blood of patients with gastric cancer. World J Surg Oncol 11: 81, 2013.

43. Qi ZX, Cai JJ, Chen LC, Yue Q, Gong Y, Yao Y and Mao Y: TRIM28 as an independent prognostic marker plays critical roles in glioma progression. J Neurooncol 126: 19-26, 2016.

44. Fitzgerald S, Sheehan KM, O'Grady A, Kenny D, O'Kennedy R, Kay EW and Kijanka GS: Relationship between epithelial and stromal TRIM28 expression predicts survival in colorectal cancer patients. J Gastroenterol Hepatol 28: 967-974, 2013.

45. Fitzgerald S, Espina V, Liotta L, Sheehan KM, O'Grady A, Cummins R, O'Kennedy R, Kay EW and Kijanka GS: Stromal TRIM28-associated signaling pathway modulation within the colorectal cancer microenvironment. J Transl Med 16: 89, 2018.

c) (i) $\odot$ This work is licensed under a Creative Commons Attribution-NonCommercial-NoDerivatives 4.0 International (CC BY-NC-ND 4.0) License. 